\title{
Approximate analytical solution of the MHD Powell-Eyring fluid flow near accelerated plate
}

\author{
Fawzia Mansour Elniel ${ }^{a, b}$, Zainal Abdul Aziz ${ }^{a, b,{ }^{*}}$, Faisal Salah ${ }^{c}$, Shaymaa Mustafa ${ }^{a, b}$ \\ ${ }^{a}$ UTM-Centre for Industrial and Applied Mathematics, Ibnu Sina Institute for Scientific and Industrial Research, Universiti Teknologi Malaysia, 81310 \\ UTM Johor Bahru, Johor, Malaysia \\ ${ }^{b}$ Department of Mathematical Sciences, Faculty of Science, Universiti Teknologi Malaysia, 81310 Johor Bahru, Johor, Malaysia \\ ${ }^{c}$ Department of Mathematics, Faculty of Science, University of Kordofan, Elobid, 51111, Sudan
}

*Corresponding author: zainalaz@utm.my, zainalabdaziz@gmail.com

\section{Article history}

Received 19 October 2017

Accepted 8 November 2017

\begin{abstract}
In this article, the non-linear equation of unsteady flow of Powell-Eyring fluid is solved by using Adomian Decomposition Method (ADM). The fluid is assumed to be flowing under the effect of magnetic field. The model is developed for the case of constant accelerated plate. Sensitivity analysis is performed to show the effects of material parameters on the velocity profile and shear stress at the wall. The results confirmed the suitability of ADM in solving nonlinear equations.
\end{abstract}

Keywords: Powell-Eyring fluid, magnetohydrodynamic (MHD), unsteady flow, Adomian Decomposition Method (ADM).

\section{INTRODUCTION}

Fluids that change viscosity due to the applied stress or forces are known by non-Newtonian fluid. There are several daily applications of the non-Newtonian fluids presented in many fields such as petroleum industries, foods manufacturing, geophysics, polymer and chemical industries. Different mathematical models are developed to investigate the behaviour of these fluids [1-4]. The complicated relationship between the shear stress and rate of strain in the nonNewtonian fluids led to use many constitutive equations by authors to model the non-Newtonian fluid. The complexity and variety of these constitutive equations makes obtaining the exact solutions of nonNewtonian fluids are difficult.

Different solutions are obtained in literature for the flow induced by constant acceleration plate for different kind of fluids such as Oldroyed-B, Maxwell and second grade fluid [5, 6]. Only a limited number of these solutions are exact. The most popular approximate analytical methods that were used in literature to solve steady and unsteady flow of non- Newtonian fluids are: Adomian decomposition method (ADM) [7, 8] and Homotopy perturbation method (HPM) [9]. However, due to the difficulty of obtaining the exact solution for these fluids, a numerical methods take a part besides the approximate analytical methods [10,11].

Obtaining a solution of the non-Newtonian fluids is useful in determination of the experimental data. One of the popular non Newtonian fluids is the Powell-Eyring fluid which is derived from the kinetic theory of liquids rather than the empirical relations [12]. Sirohi et al. [13] studied the flow of Powell-Eyring fluid that induced by accelerated plate numerically by using three methods. One of these methods is based on transformation of the boundary value problem to initial value problem. Khan et al. [14] investigated the flow of PowellEyring fluid under the effect of magnetic field. Their study becomes the basis of numerous scientific and engineering applications. Recently, constitutive equations with fractional derivative have been approved to be effective tools and can be used to describe the viscoelastic properties of non-Newtonians fluids. In particular, replacing the time ordinary derivatives of stress and strain by derivatives of fractional order provides a new fractional dimensionless quantity and variable. Khan et al. [15] used the Fourier sine transform to investigate the transient flow of an Oldroyd-B fluid in a porous medium. In 2009, the fractional calculus approach is used to formulate the constitutive relationship of this viscoelastic fluid [16, 17]. The results of the two previous studies show a good match of experimental data with model results especially when the constitutive equation with the fractional derivative is used. This result motivated some researches to apply the dimensionless quantity obtained by using the fractional derivative to some other problems that have similar flow situation [18, 19]. In this work, we apply the same procedure implemented by Khan et al. [16] to find the approximate analytical solution for the flow of the Powell-Eyring fluid under the effect of MHD by using Adomian Decomposition Method.

\section{Description Of The Problem}

Let us consider the laminar unsteady flow of an incompressible Powell-Eyring fluid over the flat plate under the effect of hydromagnetic force. Assume that the fluid is bounded by an infinite plate at $y=0$. Initially the plate at rest and at $t>0$ the plate starts to move parallel to itself with uniform velocity $\mathbf{u}$. The coordinate 
system is Cartesian coordinate where the $x$ - axis parallel to the plates and $y$-axis is taken normal to it (see Fig. 1). The applied magnetic field $B_{0}$ is parallel to the $y$-axis. The induced electric field is negligible since the Reynolds number is small. Consequently, the Lorentz force $J \times B$ under these conditions is equal to $-\sigma B_{0}^{2} \mathbf{u}$, where $\sigma$ is the electrical conductivity and $J$ is the current density. The pressure $p$ is assumed to be standard atmospheric.

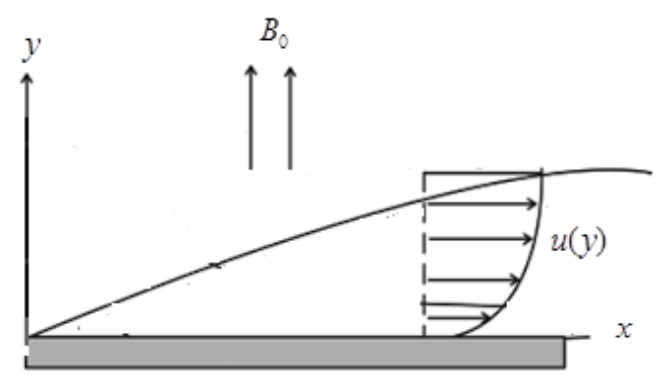

Figure 1 Geometry of the problem.

\section{Mathematical Formulation}

The balance of mass and momentum equations under the effect of magnetic field are given by

$$
\begin{aligned}
& \nabla . \mathbf{u}=0 \\
& \rho^{\lceil}\left[\frac{d \mathbf{u}}{d t}+(\mathbf{u} \cdot \nabla) \mathbf{u}\right]=\operatorname{div} \mathbf{T}+J \times B
\end{aligned}
$$

where $\rho$ is the density, $\mathbf{u}$ is the velocity vector and $d / d t$ is the material time derivative. The Cauchy stress tensor $\mathbf{T}$ of PowellEyring fluid is given by:

$$
\mathbf{T}=-p \mathbf{I}+\boldsymbol{\tau}_{x y}
$$

where $\boldsymbol{\tau}_{x y}$ is the extra stress tensor. The velocity and the stress are given as follows:

$$
\mathbf{u}=u(y, t) i \quad, \boldsymbol{\tau}=\tau(y, \mathrm{t})
$$

By substituting the values of $\mathbf{u}$ and $\boldsymbol{\tau}_{x y}$ in Equations (1) and (2), the continuity Equation (1) is satisfied while Equation (2) is reduced to

$$
\rho \frac{\partial \mathbf{u}}{\partial t}=-\frac{\partial p}{\partial x}+\frac{\partial \tau_{y x}}{\partial y}-\sigma B_{0}^{2} \mathbf{u}
$$

The extra stress tensor $\boldsymbol{\tau}_{x y}$ of Powell-Eyring fluid model is given by Powell and Eyring [12] :

$$
\tau_{y x}=\mu \frac{\partial \mathbf{u}}{\partial y}+\frac{1}{\beta} \operatorname{Sinh}^{-1}\left(\frac{1}{c} \frac{\partial \mathbf{u}}{\partial y}\right)
$$

where $\beta, c$ are the material parameters and $\mu$ is the dynamic viscosity. The Taylor expansion of the inverse hyperbolic term in the above equation is given by $[20,21]$ :
$\operatorname{Sinh}^{-1}\left(\frac{1}{c} \frac{\partial \mathbf{u}}{\partial y}\right) \cong \frac{1}{c} \frac{\partial \mathbf{u}}{\partial y}-\frac{1}{6}\left(\frac{1}{c} \frac{\partial \mathbf{u}}{\partial y}\right)^{3},\left|\frac{1}{c} \frac{\partial \mathbf{u}}{\partial y}\right| \ll 1$

Substitute Equation (7) into Equation (6) to get $\tau_{x x}=\tau_{y y}=\tau_{x z}=\tau_{z z}=\tau_{y z}=0$ and

$$
\tau_{y x}=\left[\mu+\frac{1}{\beta c}\right] \frac{\partial \mathbf{u}}{\partial y}-\frac{1}{6 c^{3} \beta}\left(\frac{\partial \mathbf{u}}{\partial y}\right)^{3}
$$

\section{Flow due to constant accelerated plate}

Assuming that the body force $\frac{\partial p}{\partial x}=0$ then by using Equations (8) and (4) into Equation (5) we get:

$$
\frac{\partial \mathbf{u}}{\partial t}=\left[v+\frac{1}{\beta \rho c}\right] \frac{\partial^{2} \mathbf{u}}{\partial y^{2}}-\frac{1}{2 \rho c^{3} \beta} \frac{\partial^{2} \mathbf{u}}{\partial y^{2}}\left(\frac{\partial \mathbf{u}}{\partial y}\right)^{2}-\frac{\sigma B_{0}^{2} \mathbf{u}}{\rho}
$$

The corresponding initial and boundary conditions for Equation (9) in the case of constant accelerated plate are

$u(y, 0)=0, \frac{\partial u(y, t)}{\partial t}=0$, when $\mathrm{y}>0$

$u(0, t)=A t \quad$ for $\quad t>0$

we introduced the following dimensionless variable as given in [17-19]

$\tau=t\left(\frac{A^{2}}{v}\right)^{\frac{1}{3}}, \xi=\mathrm{y}\left(\frac{A}{v^{2}}\right)^{\frac{1}{3}}, \quad \mathrm{G}=\frac{u}{(A v)^{\frac{1}{3}}}$

the governing problem in dimensionless form takes the form

$\frac{\partial G(\xi, \tau)}{\partial \tau}+M G(\xi, \tau)=\alpha \frac{\partial^{2} G(\xi, \tau)}{\partial \xi^{2}}-\gamma \frac{\partial^{2} G(\xi, \tau)}{\partial \xi^{2}}\left(\frac{\partial G(\xi, \tau)}{\partial \xi}\right)^{2}, \quad \xi, \tau>0$

And the boundary conditions take the form

$G(0, \tau)=\tau, \quad G(\infty, \tau)=0, \quad \tau>0$,

$G(\xi, 0)=0, \quad \frac{\partial G(\xi, 0)}{\partial \tau}=0, \quad \xi>0$,

Where

$\alpha=\left[1+\frac{1}{v c \beta \rho}\right], \mathrm{M}=\frac{\sigma B_{0}^{2}}{\rho}\left(\frac{v}{A^{2}}\right)^{\frac{1}{3}}, \gamma=\frac{1}{2 \rho \beta c^{3}}\left(\frac{A^{4}}{v^{5}}\right)$ 
Here $M$ is the non-dimensional magnetic parameter, $\alpha$ and $\gamma$ are the non-dimensional non- Newtonian parameters.

\section{Solution for constant accelerated plate}

Adomian decomposition method (ADM) is developed by Adomian [22] to solve the nonlinear equations by using the iterative technique to find the series solution. The method is applicable to a wide class of nonlinear partial di erential equations, and integral equations. To understand the basic concept of ADM, we write the differential equation in operator form as follows:

$$
\begin{aligned}
& L_{\tau} u(\xi, \tau)+L_{\xi} u(\xi, \tau)+R u(\xi, \tau)+N u(\xi, \tau)=g(\xi, \tau) \\
& L_{\xi} u(\xi, \tau)=g(\xi, \tau)-L_{\tau} u(\xi, \tau)-R u(\xi, \tau)-N u(\xi, \tau)
\end{aligned}
$$

where $L_{\xi}=\frac{\partial^{2}}{\partial \xi^{2}}$ and $L_{\tau}=\frac{\partial}{\partial \tau}$ are linear invertible operators, $g(\xi, \tau)$ is the source term, $R u(\xi, \tau)$ is the remaining linear term and $N u(\xi, \tau)$ is the non-linear term which can be expanded by using the Adomian polynomial $A_{n}$. If we apply the inverse operator $L_{\xi}^{-1}=\iint(). d \xi d \xi$ to Equation (18) yields

$L_{\xi}^{-1} L_{\xi} u(\xi, \tau)=L_{\xi}^{-1} g(\xi, \tau)-L_{\xi}^{-1} L_{\tau} u(\xi, \tau)-L_{\xi}^{-1} R u(\xi, \tau)-L_{\xi}^{-1} N u(\xi, \tau)$

The integration of the left hand side in Equation (19) gives

$$
L_{\xi}^{-1} L_{\xi} u(\xi, \tau)=u(\xi, \tau)-u(0, \tau)-\xi u_{\xi}(0, \tau)
$$

According to ADM the unknown function $u(\xi, \tau)$ is decomposed into its components where the series solution $u(\xi, \tau)$ is defined by:

$u(\xi, \tau)=\sum_{n=0}^{\infty} u_{n}(\xi, \tau)=u_{0}+u_{1}+u_{2}+\ldots \ldots$

Assume the non-linear term in Equation (19) $N u(\xi, \tau)=\sum_{n=0}^{\infty} A_{n}$.

By using the boundary condition and substition of Equations (20) and (21) into Equation (19) yields:

$$
\begin{aligned}
& u_{0}+u_{1}+\ldots=f(\xi, \tau)-L_{\xi}^{-1} L_{\tau}\left[u_{0}+u_{1}+\ldots\right] \\
& -L_{\xi}^{-1} R\left[u_{0}+u_{1}+\ldots\right]-L_{\xi}^{-1}\left[A_{0}+A_{1}+\ldots\right]
\end{aligned}
$$

where $f(\xi, \tau)=\phi-L_{\xi}^{-1} g(\xi, \tau)$ is the zero component. Consequently, the recursive relation is defined as:

$$
\begin{aligned}
& u_{0}=f(\xi, \tau) \\
& u_{1}=-L_{\xi}^{-1} L_{\tau}\left[u_{0}\right]-L_{\xi}^{-1} R\left[u_{0}\right]-L_{\xi}^{-1}\left[A_{0}\right] \\
& u_{2}=-L_{\xi}^{-1} L_{\tau}\left[u_{1}\right]-L_{\xi}^{-1} R\left[u_{1}\right]-L_{\xi}^{-1}\left[A_{1}\right]
\end{aligned}
$$

$\cdots$

Rewrite Equation (13) in operator form as in Equation (22) and divide by $\alpha$ yields

$$
\begin{aligned}
& L_{\xi}[G(\xi, \tau)]=\frac{1}{\alpha} L_{\tau}[G(\xi, \tau)]+\frac{M}{\alpha} G(\xi, \tau) \\
& +\frac{\gamma}{\alpha} \frac{\partial^{2} G(\xi, \tau)}{\partial \xi^{2}}\left(\frac{\partial G(\xi, \tau)}{\partial \xi}\right)^{2},
\end{aligned}
$$

we followed the same procedure described from Equations (19) - (22) to find the components of the solution. Since the initial condition in Equation (15) is equal to zero, then the operator related to $\tau$ cannot be applied. Consequently, the linear invertible operator in $\xi$ direction is implemented following Wazwaz [23] and Gul et al. [24]. Apply the inverse operator to both sides of Equation (24) where $L_{\xi}^{-1}=\iint(). d \xi d \xi$, and use the boundary conditions (15) we get:

$$
\begin{aligned}
& G(\xi, \tau)=\tau+h(\tau)+\frac{1}{\alpha} L_{\xi}^{-1}\left[L_{\tau} G(\xi, \tau)\right]+\frac{M}{\alpha} L_{\xi}^{-1}[G(\xi, \tau)] \\
& +\frac{\gamma}{\alpha} L_{\xi}^{-1}\left[\frac{\partial^{2} G(\xi, \tau)}{\partial \xi^{2}}\left(\frac{\partial G(\xi, \tau)}{\partial \xi}\right)^{2}\right]
\end{aligned}
$$

Where $\xi h(\tau)=\xi u_{\xi}(0, \tau)$, the non-linear term

$N=\frac{\partial^{2} G(\xi, \tau)}{\partial \xi^{2}}\left(\frac{\partial G(\xi, \tau)}{\partial \xi}\right)^{2}$ is represented by the Adomian's polynomials $N=\sum_{n=0}^{\infty} A_{n}=A_{0}+A_{1}+\ldots \ldots$ which are calculated as follows:

$A_{0}=\left(\frac{d G_{0}}{d \xi}\right)^{2} \frac{d^{2} G_{0}}{d \xi^{2}}$

$A_{1}=\left(\frac{d G_{0}}{d \xi}\right)^{2} \frac{d^{2} G_{1}}{d \xi^{2}}+2 \frac{d G_{0}}{d \xi} \frac{d G_{1}}{d \xi} \frac{d^{2} G_{0}}{d \xi^{2}}$

$A_{2}=\left(\frac{d G_{1}}{d \xi}\right)^{2} \frac{d^{2} G_{0}}{d \xi^{2}}+2 \frac{d G_{0}}{d \xi} \frac{d G_{1} d^{2} G_{1}}{d \xi d \xi^{2}}+2 \frac{d G_{0}}{d \xi} \frac{d G_{2}}{d \xi} \frac{d^{2} G_{0}}{d \xi^{2}}+\left(\frac{d G_{0}}{d \xi}\right)^{2} \frac{d^{2} G_{2}}{d \xi^{2}}$.

Choose the zeroth component problem for the MHD of Powell-Eyring fluid as

$G_{0}(\xi, \tau)=\tau+\xi h(\tau)$

subject to the boundary conditions

$G(0, \tau)=\tau, \quad \tau>0$,

$G(\xi, 0)=0, \quad \frac{\partial G(0, \tau)}{\partial \tau}=1, \quad \xi>0$,

The remaining components can be written as

$G_{k+1}(\xi, \tau)=\frac{1}{\alpha} L_{\xi}^{-1}\left[L_{\tau} \sum_{n=0}^{\infty} G_{n}(\xi, \tau)\right]+\frac{M}{\alpha} L_{\xi}^{-1}\left[\sum_{n=0}^{\infty} G_{n}(\xi, \tau)\right]+\frac{\gamma}{\alpha} L_{\xi}^{-1}\left[\sum_{n=0}^{\infty} A_{n}\right], k \geq 0$

To find the first component of the solution substitute $k=0$ into Equations (28) yield: 
$G_{1}(\xi, \tau)=\frac{1}{\alpha} L_{\xi}^{-1}\left[L_{\tau} G_{0}(\xi, \tau)\right]+\frac{M}{\alpha} L_{\xi}^{-1}\left[G_{0}(\xi, \tau)\right]+\frac{\gamma}{\alpha} L_{\xi}^{-1}[A$

Similarly we can find $G_{2}, G_{3}, \ldots$

operating with the inverse linear operator $L_{\xi}^{-1}=\iint(). d \xi d \xi$, we obtain the first several component solutions, and the solution in the series form as follows

$G(\xi, \tau)=\tau+\frac{M \xi^{4}}{24 \alpha^{2}}+\frac{M^{2} \xi^{4} \tau}{24 \alpha^{2}}+\frac{M \xi^{2} \tau}{2 \alpha}+\frac{M^{2} \xi^{6}}{420 \alpha^{3}}+$

$\frac{M^{3} \xi^{6} \tau}{720 \alpha^{3}}+\left(\frac{\gamma M \xi^{3}}{6 \alpha^{2}}+\frac{\gamma M^{2} \xi^{5}}{15 \alpha^{3}}\right) h(\tau)^{3}+\frac{\gamma^{2} \xi^{3} h(\tau)^{5}}{6 \alpha^{3}} \ldots$

To complete the determination of the series solution we should determine the function

$h(\tau)=u_{\xi}(0, \tau)$ which is not given ,with the help of equation (14)

substitute $\tau=0$ and equating the coefficient of same power in the two sides of Equation (30) it is found that the series solution is:

$G(\xi, \tau)=\tau+\frac{(1+M \tau) \xi^{2}}{2 \alpha}+\frac{M(2+M \tau) \xi^{4}}{24 \alpha^{2}}+$
$\frac{M^{2}(3+M \tau) \xi^{6}}{720 \alpha^{3}}+\frac{M^{3}(4+M \tau) \xi^{8}}{40320 \alpha^{4}}+\ldots$

The direct calculation shows that $G(\xi, \tau)$ satisfied Equations (13) -

(15) when $\gamma=0$. Calculating more terms of Equation (28) produced more accurate solution.

\section{RESULTS AND DISCUSSION}

In the present work we have considered the unsteady flow of an incompressible, isothermal, and homogeneous Powell-Eyring fluid. The governing partial differential equation for the constant accelerated flow of Powell-Eyring fluid has been analytically solved by using Adomian Decomposition Method. In Figures 2 and 3, the velocity $G(\xi, \tau)$ is plotted versus $\xi$ with different non-Newtonian parameters to highlight the effect of these parameters on the constant accelerated flow.

Figure (2) shows the variation of the velocity $G(\xi, \tau)$ by changing the time $\tau$ for different values of $\xi$.Generally there is a proportional relationship between $\tau$ and $G(\xi, \tau)$. Moreover, it is noticed that at fixed value of $\xi$, the velocity increases by constant value when time is changed from 0.2 to 1 . Figure 3 illustrates the influence of the non-Newtonian fluid parameter $\alpha$ on the velocity. It is found that the velocity $G(\xi, \tau)$ increasing with decreasing the nonNewtonian parameter $\alpha$. This increase in velocity is due to the large value of viscosity at a small value of $\alpha$. Generally the results indicate that when the non-Newtonian parameter $\gamma$ equal zero the solution depend on the non-Newtonian parameter $\alpha$. the plot of the solution is shown to be of Newtonian behaviour when $\gamma=M=0$; see Fig 4.

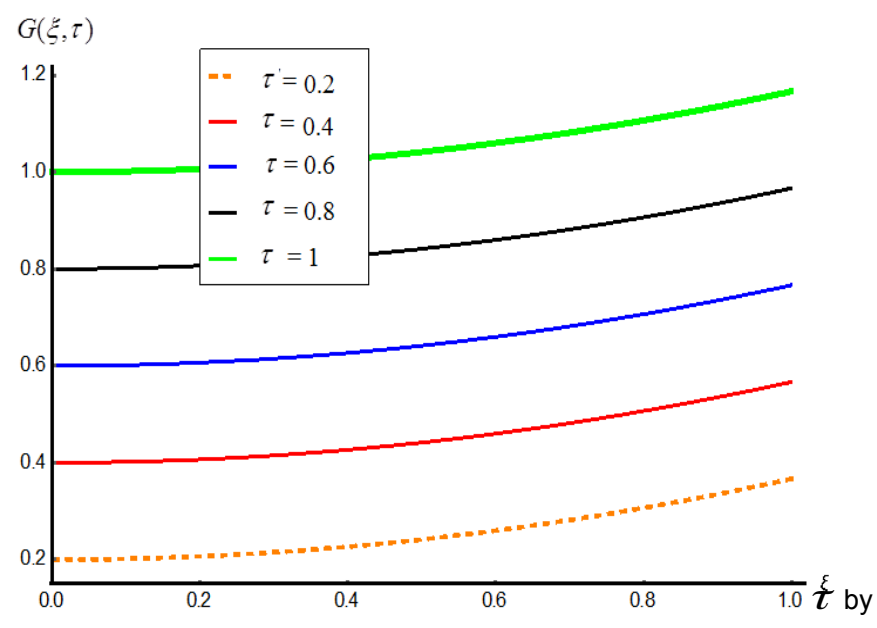

Figure 2 Velocity profile versus $\xi$ for different values of using $\alpha=3, M=0$

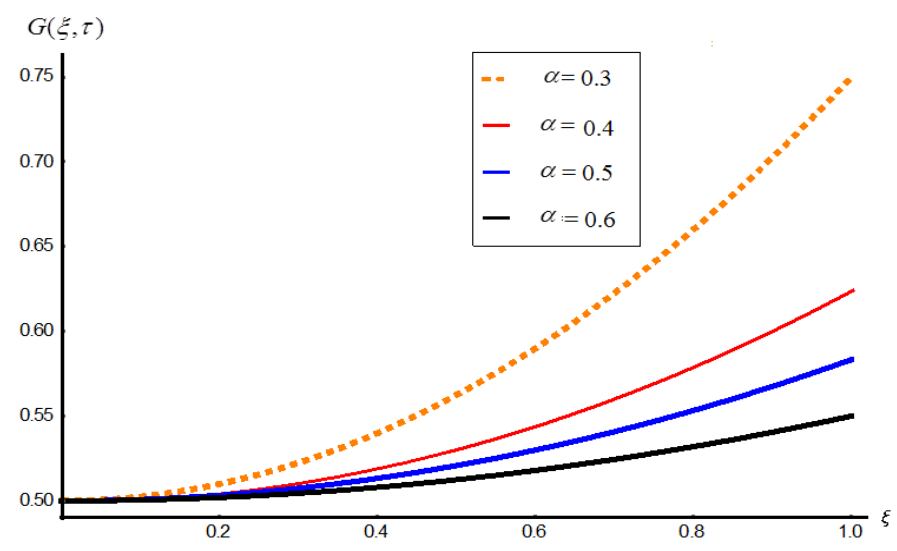

Figure 3 Velocity profile for different values of $\alpha$ by using $\gamma=0, M=0$

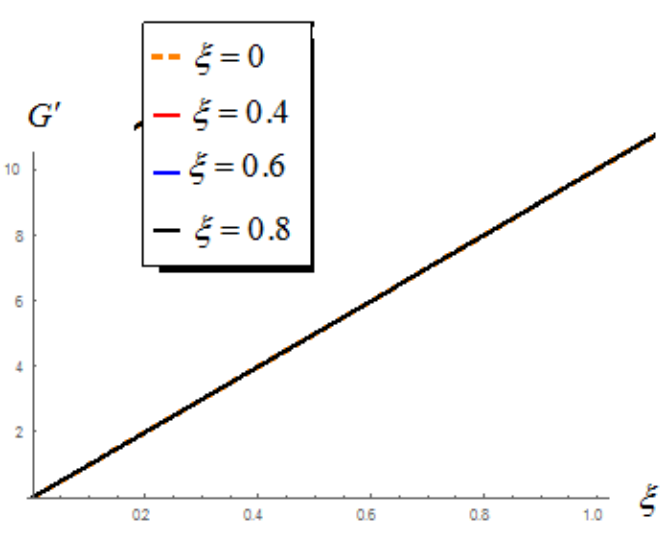

Figure 4: Velocity gradient for different values of $\xi$ by using $\gamma=M=0, \tau=0.1$ 


\section{CONCLUSION}

The series solution for the velocity of MHD unsteady flow of Powell-Eyring fluid was obtained using the ADM. Generally, several methods are used in solving related non-linear PDEs. The Adomian Decomposition Method is one of the effective methods, which provides an efficient solution for the physical equations modelled by nonlinear differential equations. The implementation of ADM and the calculation of Adomian polynomials do not need complex calculations, except for simple and elementary operations effectively used. This has led to reduce the computational work compared to the other methods. Furthermore, the influences of the parameters in the velocity have been shown in different graphs. It was observed that the velocity field depends on the involved parameters. In addition, it was noticed that decreasing the value of non-Newtonian parameters increased the velocity flow of the fluid. Moreover, it was found that the shear thinning effect in the fluid was higher when the value of $\alpha$ decreased.

\section{ACKNOWLEDGEMENT}

The authors thankfully acknowledged the financial support from Ministry of Higher Education (MOHE), Malaysia and FRGS Project Vot No: 4F354.

\section{REFERENCES}

[1] Khan, I., Malik, M., Salahuddin, T., Khan, M., and Rehman, K. U. 2017. Homogenous-heterogeneous reactions in MHD flow of Powell-Eyring fluid over a stretching sheet with Newtonian heating. Neural Computing and Applications. 1-8.

[2] Hayat, T., Iqbal, Z., Qasim, M., and Obaidat, S. 2012. Steady flow of an Eyring Powell fluid over a moving surface with convective boundary conditions. International Journal of Heat and Mass Transfer. 55(7), 1817-1822.

[3] Fetecau, C., Zierep, J., Bohning, R., and Fetecau, C. 2010. On the energetic balance for the flow of an Oldroyd-B fluid due to a flat plate subject to a time-dependent shear stress. Computers \& Mathematics with Applications. 60(1), 74-82.

[4] Ishak, A., Nazar, R., and Pop, I. 2006. Mixed convection boundary layers in the stagnation-point flow toward a stretching vertical sheet. Meccanica. 41(5), 509-518.

[5] Fetecau, C., Prasad, S. C., and Rajagopal, K. R. A 2007. note on the flow induced by a constantly accelerating plate in an Oldroyd-B fluid. Applied Mathematical Modelling. 31(4), 647-654.

[6] Jamil, M., Rauf, A., Fetecau, C., and Khan, N. Helical flows of second grade fluid due to constantly accelerated shear stresses. Communications in Nonlinear Science and Numerical Simulation. 16(4), 1959-1969.

[7] Adesanya, S. O., Falade, J. A., and Rach, R. 2015. Effect of couple stresses on hydromagnetic Eyring-Powell fluid flow through a porous channel. Theoretical and Applied Mechanics. 42(2), 135-150.
[8] Siddiqui, A., Haroon, T., and Zeb, M. 2014. Analysis of Eyring-Powell fluid in helical screw rheometer. The Scientific World Journal. 382(2015), 355-358.

[9] Zaman, H., Zaman, H., and Zaman, H. 2013. for the Eyring-Powell model with porous walls Unsteady incompressible Couette flow problem. American Journal of Computational Mathematics. 3(4), 313-325.

[10] Akbar, N. S., Ebaid, A., and Khan, Z. 2015. Numerical analysis of magnetic field effects on Eyring-Powell fluid flow towards a stretching sheet. Journal of Magnetism and Magnetic Materials. 382, 355-358.

[11] Ellahi, R., Shivanian, E., Abbasbandy, S., and Hayat, T. 2016. Numerical study of magnetohydrodynamics generalized Couette flow of EyringPowell fluid with heat transfer and slip condition. International Journal of Numerical Methods for Heat \& Fluid Flow. 26(5), 1433-1445.

[12] Powell, R. E. and Eyring, H. 1944. Mechanism for relaxation theory of viscosity. Nature. 154(55), 427-428.

[13] Sirohi, V., Timol, M., and Kalthia, N. 1987. Powell-Eyring model flow near an accelerated plate. Fluid Dynamics Research. 2(3), 193-204.

[14] Khan, N. A., Aziz, S., and Khan, N. A. 2014. MHD flow of PowellEyring fluid over a rotating disk. Journal of the Taiwan Institute of Chemical Engineers. 45(6), 2859-2867.

[15] Khan, M., Saleem, M., Fetecau, C., and Hayat, T. 2007. Transient oscillatory and constantly accelerated non-Newtonian flow in a porous medium. International Journal of Non-Linear Mechanics. 42(10), 12241239.

[16] Khan, M., Ali, S. H., and Qi, H. 2009. Some accelerated flows for a generalized Oldroyd-B fluid. Nonlinear analysis. Real World Applications. 10(2), 980-991.

[17] Khan, M., Ali, S. H., and Qi, H. 2009. On accelerated flows of a viscoelastic fluid with the fractional Burgers' model. Nonlinear Analysis Real World Applications. 10(4), 2286-2296.

[18] Aziz, Z. A., Salah, F., and Ching, D. L. C. 2011. On accelerated flow for MHD generalized burgers' fluid in a porous medium and rotating frame. IAENG International Journal of Applied Mathematics. 41(3), 199-205.

[19] Khan, I., Ali, F., Mustapha, N., and Shafie, S. 2015. Closed-form solutions for accelerated MHD flow of a generalized Burgers' fluid in a rotating frame and porous medium. Boundary Value Problems. 2015(8), $1-17$.

[20] Jalil, M., Asghar, S., and Imran, S. 2013. Self similar solutions for the flow and heat transfer of Powell-Eyring fluid over a moving surface in a parallel free stream. International Journal of Heat and Mass Transfer. $65,73-79$.

[21] Hansen, A. and Na, T. Y. 1986. Similarity solutions of laminar, incompressible boundary layer equations of non-Newtonian fluids. Journal of Basic Engineering. 90(1), 71-74.

[22] Adomian, G. 1991. Solving frontier problems modelled by nonlinear partial differential equations. Computers \& Mathematics with Applications. 22(8), 91-94.

[23] Wazwaz, A.-M. 2010. Partial Differential Equations and Solitary Waves Theory. Springer Science \& Business Media.

[24] Gul, T., Ghani, F., Islam, S., Shah, R. A., Khan, I., Nasir, S., and Sharidan, S. 2016. Unsteady thin film flow of a fourth grade fluid over a vertical moving and oscillating belt. Propulsion and Power Research. $5(3), 223-235$. 